\title{
Cognitive Impairments and Associated Structural Brain Changes in Metabolic Syndrome and Implications of Neurocognitive Intervention
}

\author{
Parastou Kordestani-Moghadam ${ }^{1}$, Shervin Assari' ${ }^{2}$, Sarah Nouriyengejeh ${ }^{3}$, Fatemeh Mohammadipour ${ }^{4}$, Ata Pourabbasi ${ }^{3, *}$ \\ 'Social Determinants of Health Research Center, Lorestan University of Medical Science, Khorramabad, Iran; ${ }^{2}$ Department of Psychiatry, University of Michigan, Ann \\ Arbor, MI, USA; ${ }^{3}$ Endocrinology and Metabolism Research Center, Endocrinology and Metabolism Clinical Sciences Institute, Tehran University of Medical Sciences, \\ Tehran, Iran; ${ }^{4}$ Student Research Committee, Lorestan University of Medical Science, Khorramabad, Iran
}

Currently, metabolic syndrome has become a global health problem. Alterations in neurocognitive functions among patients with metabolic syndrome are important issues in this disorder. In this paper, studies on metabolic syndrome were reviewed and their importance emphasized for the benefit of experts and policy makers. Metabolic syndrome activates inflammatory mediators that disrupt brain metabolism. These mediators can be activated by metabolic inflammation and microvascular disorders and may further cause damage to the white matter and impair cognitive function. These alterations can result in serious changes in cognitive abilities. The association between cognitive changes and metabolic syndrome has been independently evaluated in several studies. In addition, some areas of research in the field of metabolic syndrome include the effectiveness of neurocognitive interventions to enhance normal behaviors or reduce risky behaviors in patients. Structural brain correlates of health-related behaviors provide a basis for designing more effective behavioral interventions by identifying the corresponding brain regions and using behavioral interventions.

Key words: Brain structure, Metabolic syndrome, Neurocognitive intervention

\author{
Received March 15, 2020 \\ Reviewed March 29, 2020 \\ Accepted April 26, 2020 \\ ${ }^{*}$ Corresponding author \\ Ata Pourabbasi \\ (i) \\ https://orcid.org/0000-0002-9500-640X \\ Endocrinology and Metabolism Research \\ Center, Endocrinology and Metabolism \\ Clinical Sciences Institute, Tehran \\ University of Medical Sciences, Ground \\ Floor, EMRI Central Building, \\ Al-E-Ahmad highway, \\ Tehran 1411713137, Iran \\ Tel: +98-2188220091 \\ Fax: +98-2188220069 \\ E-mail: atapoura@gmail.com
}

\section{INTRODUCTION}

Metabolic syndrome, a non-communicable disease (NCD), has become a global health problem. The World Health Organization has cited the metabolic syndrome as a highly prevalent disease. ${ }^{1}$ Metabolic syndrome is a collection of clinical and laboratory abnormalities that cause many problems in an individual and is associated with an increased risk of NCDs such as cardiovascular disease and diabetes mellitus. In addition to the health problems, metabolic syndrome also lowers the quality of life and increases economic burden. The risk factors for metabolic syndrome can cause health problems. The worldwide prevalence of this disease in the elderly ranges from $20 \%$ to $25 \%$. Nearly $20 \%$ of residents in western Iran and $30 \%$ of Tehran residents suffer from metabolic syndrome. Obesity, a risk factor for metabolic syndrome, causes the death of 100,000 Americans annually. ${ }^{2-4}$ Other challenges include the incidence of obesity and diabetes mellitus in children and adolescents, which has recently become a health concern., ${ }^{5,6}$

Currently, neurocognitive dysfunctions in patients with metabolic syndrome is an important issue associated with the disorder, however the issue has not been well addressed. ${ }^{6,7}$ Therefore, in the present study, a literature search was performed to determine the

Copyright (C) 2020 Korean Society for the Study of Obesity

(a) This is an Open Access article distributed under the terms of the Creative Commons Attribution Non-Commercial License (https://creativecommons.org/licenses/by-nc/4.o/) which permits unrestricted non-commercial use, distribution, and reproduction in any medium, provided the original work is properly cited. 
structural brain changes in metabolic syndrome patients. Finally, after selecting the relevant studies, the relationship between metabolic systems, neurocognitive impairment, and brain structure in metabolic syndrome were the areas of particular focus. Brain mapping studies were used for confirmation of findings.

\section{BRAIN STRUCTURAL CHANGES IN METABOLIC SYNDROME}

Mediators associated with metabolic syndrome are correlated with pathways that activate metabolic inflammation and microvascular disorders and may further cause damage to the brain white matter and reduce cognitive function (Fig. 1). ${ }^{8}$ Many complications of metabolic syndrome can be attributed to glucose fluctuation, an important indicator of the disorder. Insulin resistance is a serious condition in metabolic syndrome which can cause fluctuations in blood glucose and increase serum insulin levels in the af- fected patients. It is well-known that insulin resistance can alter insulin signaling in the brain, and with oxidative stress, can cause glycation of the brain tissue and lead to brain inflammation. ${ }^{2}$

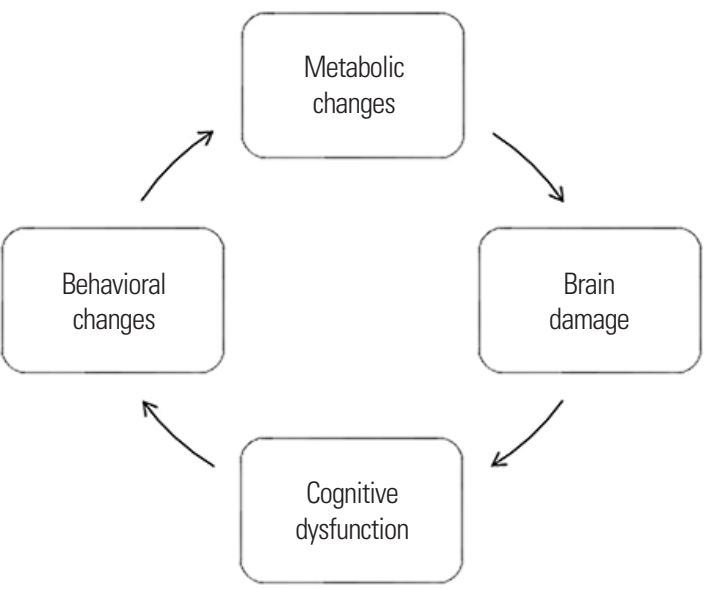

Figure 1. Metabolic changes, brain damage, cognitive dysfunction, and behavioral changes are in a cyclic relationship and any change in one part can affect the others.

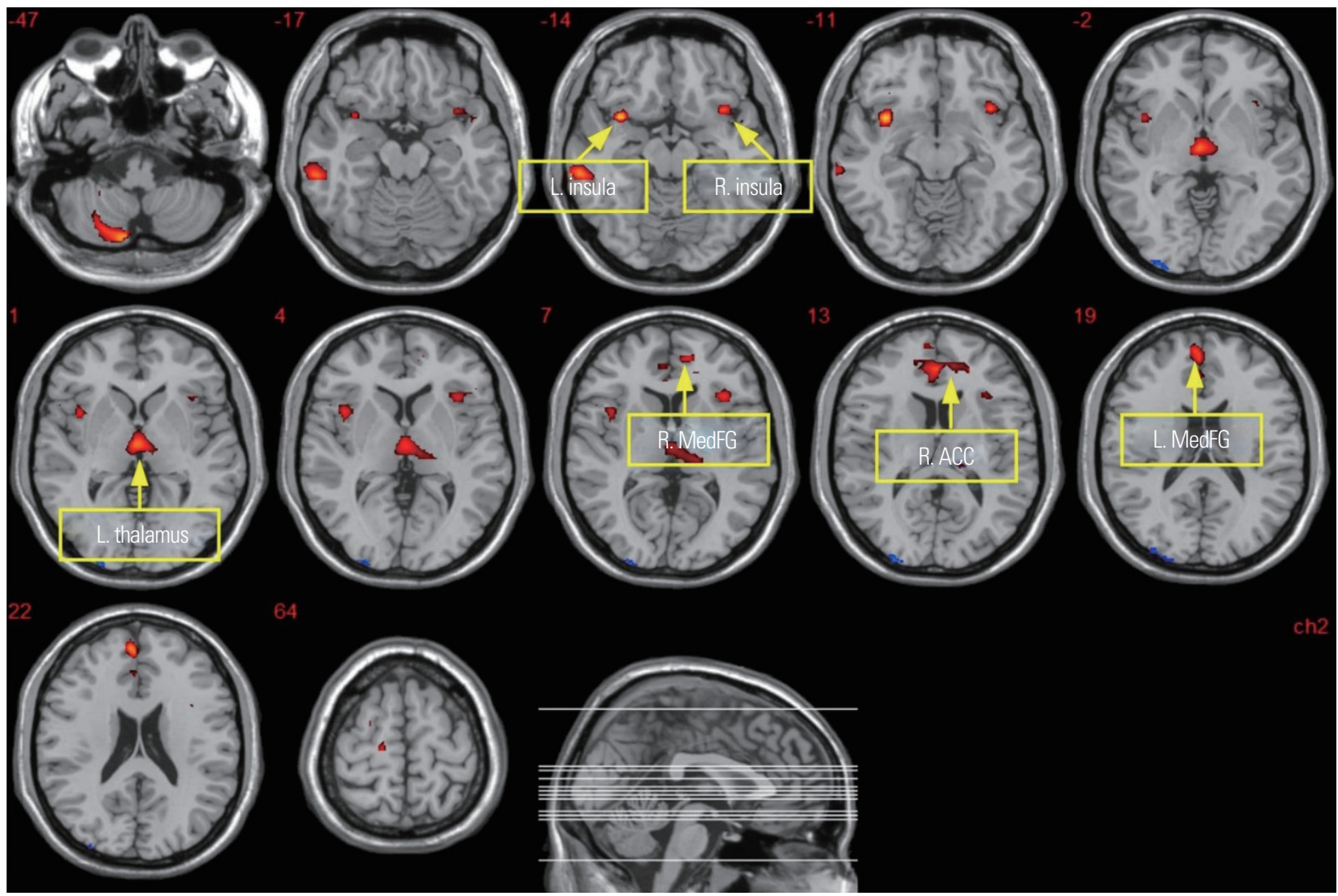

Figure 2. The red regions are reductions in grey matter volume in obese patients versus normal weight patients. The blue regions are increases in grey matter volume in obese patients versus normal weight patients. L, left; $R$, right; MedFG, medial frontal gyrus; $A C C$, anterior cingulate cortex ( $P=0.005$, cluster size $>50$ ). Reprinted from Wang H, et al. Sci Rep 2017;7:40595. ${ }^{9}$ 
A high glucose level in the brain associated with metabolic syndrome is observed in a variety of cognitive dysfunction including dementia. In addition, obesity and hypertension are considered modifiable factors in dementia and severe degeneration of the brain (Fig. 2). ${ }^{9}$ In previous studies, the control of metabolic syndrome was shown to slow down the process of brain degeneration in both white and grey matter tissues. ${ }^{4,10}$ Metabolic syndrome was a risk factor not only for diabetes mellitus and cerebrovascular disorders but also forthe progression of Alzheimer disease. ${ }^{11}$

Furthermore, metabolic syndrome can accelerate the onset and progression of cerebral small vessel dysfunctions by causing changes in the structure and function of the blood vessels, which can lead to mild bleeding, white matter damage, and brain atrophy. ${ }^{12}$ Most of these atrophies occur in deep areas of the white matter, especially in the right frontal lobe, brain gyri, optic radiations, and dorsal cingulum bundle. $^{2}$

In addition to direct effects on brain tissue, metabolic syndrome can cause indirect damage, which has not been fully addressed. For example, sedentary lifestyle, a risk factor for metabolic syndrome, decreases oxygenation and circulation and can cause long-term damage to neural structures. Thus, brain oxygenation and elimination of toxic substances are significantly improved by changing lifestyle and promoting physical activity. This process independently can also improve brain function and subsequently, quality of life. ${ }^{6}$

\section{COGNITIVE CHANGES IN METABOLIC SYNDROME}

In addition to numerous studies conducted on the structural changes in the brain of patients with metabolic syndrome, the association between cognitive changes and metabolic syndrome has been independently evaluated in several studies. The attention and neuromuscular connections were reduced in cognitive tasks associated with the parietal and occipital lobes in elderly patients with metabolic syndrome. ${ }^{13,14}$ Based on various study results regarding metabolic syndrome effects on brain structure and consequent changes in brain tissue integrity and decrease of cognitive ability, ${ }^{4}$ obesity, hypertension, and hyperglycemia apparently have the greatest effect in reducing cognitive performance and abilities. ${ }^{2,15}$ The effects of metabolic syndrome on cognitive skills included a decreased decision-making ability, reduced memory function, and decline in the reward system, and apparently a reduction of cognitive efficiency can decrease the individual's ability in the attributed skills. ${ }^{16}$

Metabolic syndrome is not the only cause of impaired brain metabolism and the resulting cognitive and structural changes. Currently, metabolic disorders have attracted increased attention among metabolic diseases. In addition, due to the high prevalence of metabolic syndrome and diabetes mellitus, as well as the slowing down of metabolism in all organs including the brain, the importance of metabolic syndrome has increased. For example, brain metabolism plays a major role in pathogenesis of some neurological disorders such as Alzheimer's disease $\mathrm{e}^{17-19}$ by hypometabolismpromoted $\mathrm{A} \beta$ deposition. ${ }^{20}$ Furthermore, the findings based on proton magnetic resonance spectroscopy have shown the alteration of metabolite concentration in various areas of the brain in different neurometabolic disorders such as multiple sclerosis, ${ }^{21}$ brain tumors, ${ }^{22,23}$ epilepsy, ${ }^{24,25}$ Alzheimer disease, ${ }^{26-28}$ and dementia ${ }^{29}$ is significantly correlated with cognitive decline. Based on the abovementioned studies, metabolic diseases of the brain can lead to changes in the structure and function of brain areas associated with cognition; most metabolic diseases of the brain cause changes in neuronal structure and cognitive decline.

In several studies, significantly decreased grey matter volume in the cerebellum, thalamus, pre-central/post-central gyrus, middle temporal gyrus, superior parietal lobule, and increased grey matter volume in the pallidum and hippocampus were observed in patients with obesity. ${ }^{30-32}$

\section{COGNITIVE NEUROLOGICAL INTERVENTIONS IN METABOLIC SYNDROME AND THEIR EFFECTIVENESS}

In some areas of research in the field of metabolic syndrome, the effectiveness of neurocognitive interventions to enhance normal behaviors or reduce risky behaviors in patients is investigated. For example, in a study conducted by Garcia-Silva, ${ }^{33} 48$ cognitive behavioral interventions such as saying "no" effectively, anger control, and appetite control, were taught to volunteers with metabolic syndrome. After 3-6 months, biochemical indices and body mass index were remeasured and significant changes were observed in 
these indices. The main change due to the interventions was the success of individuals in following a diet for metabolic syndrome. In another study, researchers showed that cognitive-behavioral therapy over the course of 12 months had a positive effect on weight loss in obese patients. However, behavioral interventions without the involvement of cognitive methods, did not have any significant effect on weight loss. ${ }^{34}$ Another study by Augustijn et al. ${ }^{35}$ on children showed that cognitive interventions, along with other interventions, could be effective in controlling weight and binge-eating behavior. A combination of cognitive interventions and other interventions such as physical activity promotion can lead to permanent changes in the behavior, habits and lifestyle of children. The authors found a significant increase in total $(P<$ $0.001)$ and cerebellar $(P<0.001)$ grey matter in patients with obesity after the multidimensional intervention compared with healthy children.

In several studies, the relevance and effectiveness of these interventions on structural changes in the brain were investigated and the mechanisms of cognitive intervention effects were evaluated. In a study by Shigaeff et al., ${ }^{13}$ significant increases in the grey matter volume and total brain mass were observed when food choices and dietary behaviors were modified (which consists of a set of nutritional behaviors). The volunteers who participated in the study were adults with metabolic syndrome. In a meta-analysis study recently conducted, cognitive therapy significantly reduced bingeeating episodes and abstinence from binge-eating. ${ }^{36}$

\section{CONSIDERATION OF STRUCTURAL BRAIN CHANGES TO DESIGN EFFECTIVE INTERVENTIONS}

Attention to behavioral patterns in metabolic syndrome is important in two aspects. First, the behaviors associated with metabolic syndrome including high-risk behaviors such as abnormal nutritional habits and sedentary lifestyle should be addressed. Most of the interventions in this area are cognitive behavioral interventions to create true beliefs, normal behaviors, and promote healthy lifestyles. Second, the behavioral changes caused by cognitive impairments are associated with structural disorders of the brain due to metabolic syndrome. ${ }^{1,3,4}$ For example, the effects of metabolic syndrome on memory have been shown in several studies, and this cognitive impairment can lead to serious behavioral problems in patients. $^{14-16}$

Therefore, an important goal of cognitive-behavioral interventions in metabolic syndrome is the consideration of various disorders. Cognitive-behavioral interventions in both preventive and therapeutic dimensions should be added to clinical guidelines for metabolic syndrome management to prevent the behavioral disorders caused by cognitive impairments in patients. In addition, structural brain correlates of health-related behaviors provide a basis for designing more effective behavioral interventions by identifying the corresponding brain regions and using brain-targeted behavioral interventions. ${ }^{33-35}$

Consequently, large studies should be performed to fully understand the map of structural changes in the brain of subjects with metabolic syndrome, the corresponding behaviors, interventions to manage the behaviors, and prevent future structural brain disorders.

\section{CONCLUSION}

Most of the neurocognitive interventions studied in patients with metabolic syndrome have been designed to modify lifestyles, promote normal behaviors, and reduce risky behaviors, but less attention has been given to the neural capacities needed to institutionalize and maintain normal health behaviors, a subject generally studied in social neuroscience. Human behavior is coded in the brain, and the development of a specific part of the brain is needed for manifestation of human behavior. For example, some parts of the cerebral cortex such as the superior medial frontal are associated with appetite control behavior and the development of brain capacity in this area is associated with such behavior. Interventions to activate or develop this part of the cortex should be performed to provide the neural capacity needed to manifest and maintain the appetite-related behavior. Otherwise, other interventions, even if they have short-term effects, will not lead to sustainable behavior in the long-term. Therefore, in metabolic syndrome, the first step in behavioral interventions to establish sustainable behavior and lifestyle modification is to identify the neural correlates of the normal behaviors and focus on their development or activation concordant with the patient's age. Cognitomics is the evaluation of the relation- 
ship between diseases and cognitions and may provide relevant answers. $^{37}$

\section{CONFLICTS OF INTEREST}

The authors declare no conflict of interest.

\section{AUTHOR CONTRIBUTIONS}

Study concept and design: AP; drafting of the manuscript: FM and SN; critical revision of the manuscript: SA and PKM; administrative, technical, or material support: $\mathrm{SN}$.

\section{REFERENCES}

1. Potenza MV, Mechanick JI. The metabolic syndrome: definition, global impact, and pathophysiology. Nutr Clin Pract 2009; 24:560-77.

2. Alfaro FJ, Gavrieli A, Saade-Lemus P, Lioutas VA, Upadhyay J, Novak V. White matter microstructure and cognitive decline in metabolic syndrome: a review of diffusion tensor imaging. Metabolism 2018;78:52-68.

3. Azadbakht L, Mirmiran P, Esmaillzadeh A, Azizi F. Dairy consumption is inversely associated with the prevalence of the metabolic syndrome in Tehranian adults. Am J Clin Nutr 2005;82: 523-30.

4. Mellendijk L, Wiesmann M, Kiliaan AJ. Impact of nutrition on cerebral circulation and cognition in the metabolic syndrome. Nutrients 2015;7:9416-39.

5. Pourabbasi A, Tehrani-Doost M, Ebrahimi Qavam S, Farzami J, Larijani B. Evaluation of cognitive functions in Iranian children and adolescents with diabetes mellitus. Acta Med Iran 2017; $55: 381-8$.

6. Qavam SE, Anisan A, Fathi M, Pourabbasi A. Study of relationship between obesity and executive functions among high school students in Bushehr, Iran. J Diabetes Metab Disord 2015;14:79.

7. Pourabbasi A, Tehrani-Doost M, Qavam SE, Arzaghi SM, Larijani B. Association of diabetes mellitus and structural changes in the central nervous system in children and adolescents: a systematic review. Diabetes Metab Disord 2017;16:10.
8. Yates KF, Sweat V, Yau PL, Turchiano MM, Convit A. Impact of metabolic syndrome on cognition and brain: a selected review of the literature. Arterioscler Thromb Vasc Biol 2012;32: 2060-7.

9. Wang H, Wen B, Cheng J, Li H. Brain structural differences between normal and obese adults and their links with lack of perseverance, negative urgency, and sensation seeking. Sci Rep 2017;7:40595.

10.Lampe L, Zhang R, Beyer F, Huhn S, Kharabian Masouleh S, Preusser S, et al. Visceral obesity relates to deep white matter hyperintensities via inflammation. Ann Neurol 2019;85:194203.

11. Cantero MD, Villa Etchegoyen C, Perez PL, Scarinci N, Cantiello HF. Bundles of brain microtubules generate electrical oscillations. Sci Rep 2018;8:11899.

12. Yaffe K. Metabolic syndrome and cognitive disorders: is the sum greater than its parts? Alzheimer Dis Assoc Disord 2007; 21:167-71.

13. Shigaeff N, Amaro E, Franco FG, Jacinto AF, Chiochetta G, Cendoroglo MS, et al. Functional magnetic resonance imaging response as an early biomarker of cognitive decline in elderly patients with metabolic syndrome. Arch Gerontol Geriatr 2017; 73:1-7.

14. Shigaeff N, Jacinto A, Chiochetta G, Cendoroglo MS, Amaro E, Ikeda $\mathrm{M}$, et al. Cognition and cerebral neurovascular coupling in the elderly with metabolic syndrome. Alzheimers Dement 2012;8:P492.

15. Joo SH, Yun SH, Kang DW, Hahn CT, Lim HK, Lee CU. Body mass index in mild cognitive impairment according to age, sex, cognitive intervention, and hypertension and risk of progression to Alzheimer's disease. Front Psychiatry 2018;9:142.

16. Alarcón G, Ray S, Nagel BJ. Lower working memory performance in overweight and obese adolescents is mediated by white matter microstructure. J Int Neuropsychol Soc 2016;22: 281-92.

17. Bero AW, Yan P, Roh JH, Cirrito JR, Stewart FR, Raichle ME, et al. Neuronal activity regulates the regional vulnerability to amyloid- $\beta$ deposition. Nat Neurosci 2011;14:750-6.

18. Jagust WJ, Mormino EC. Lifespan brain activity, $\beta$-amyloid, and Alzheimer's disease. Trends Cogn Sci 2011;15:520-6. 
19. Mosconi L. Glucose metabolism in normal aging and Alzheimer's disease: methodological and physiological considerations for PET studies. Clin Transl Imaging 2013;1:217-33.

20. Chételat G, Landeau B, Salmon E, Yakushev I, Bahri MA, Mézenge $\mathrm{F}$, et al. Relationships between brain metabolism decrease in normal aging and changes in structural and functional connectivity. Neuroimage 2013;76:167-77.

21. Sun J, Song H, Yang Y, Zhang K, Gao X, Li X, et al. Metabolic changes in normal appearing white matter in multiple sclerosis patients using multivoxel magnetic resonance spectroscopy imaging. Medicine (Baltimore) 2017;96:e6534.

22. Carlin D, Babourina-Brooks B, Davies NP, Wilson M, Peet AC. Variation of T2 relaxation times in pediatric brain tumors and their effect on metabolite quantification. J Magn Reson Imaging 2019;49:195-203.

23. Julià-Sapé M, Candiota AP, Arús C. Cancer metabolism in a snapshot: MRS(I). NMR Biomed 2019;32:e4054.

24. Dhamala E, Abdelkefi I, Nguyen M, Hennessy TJ, Nadeau H, Near J. Validation of in vivo MRS measures of metabolite concentrations in the human brain. NMR Biomed 2019;32:e4058.

25. Reddy SD, Younus I, Sridhar V, Reddy DS. Neuroimaging biomarkers of experimental epileptogenesis and refractory epilepsy. Int J Mol Sci 2019;20:220.

26. Kantarci K, Jicha GA. Development of $1 \mathrm{H}$ MRS biomarkers for tracking early predementia Alzheimer disease. Neurology 2019;9:209-10.

27. Oeltzschner G, Wijtenburg SA, Mikkelsen M, Edden RA, Barker PB, Joo JH, et al. Neurometabolites and associations with cognitive deficits in mild cognitive impairment: a magnetic resonance spectroscopy study at 7 Tesla. Neurobiol Aging 2019; 73:211-8.

28. Zhu XH, Lu M, Chen W. Advanced multinuclear magnetic resonance spectroscopy (MRS) imaging approaches for studying brain metabolism, neuroenergetics, and function. In: Iaizzo PA, editor. Engineering in medicine: advances and challenges. Cambridge: Academic Press; 2019. p. 463-91.

29. Crosson Bruce. Imaging in "healthy" aging and dementia: a bigger sandbox [Internet]. Georgia, AT: Crosson Bruce; 2018 [cited 2020 Jul 11]. Available from: https://cos.gatech.edu/ events/imaging-healthy-aging-and-dementia-bigger-sandbox

30. de Groot CJ, van den Akker EL, Rings EH, Delemarre-van de Waal HA, van der Grond J. Brain structure, executive function and appetitive traits in adolescent obesity. Pediatr Obes 2017; 12:e33-6.

31. Moreno-López L, Soriano-Mas C, Delgado-Rico E, Rio-Valle JS, Verdejo-García A. Brain structural correlates of reward sensitivity and impulsivity in adolescents with normal and excess weight. PLoS One 2012;7:e49185.

32. Ou X, Andres A, Pivik RT, Cleves MA, Badger TM. Brain gray and white matter differences in healthy normal weight and obese children. J Magn Reson Imaging 2015;42:1205-13.

33. Garcia-Silva J, Navarrete NN, Peralta-Ramírez MI, García-Sánchez A, Ferrer-González MÁ, Caballo VE. Efficacy of cognitive behavioral therapy in adherence to the mediterranean diet in metabolic syndrome patients: a randomized controlled trial. J Nutr Educ Behav 2018;50:896-904.

34. Grilo CM, Masheb RM, Wilson GT, Gueorguieva R, White MA. Cognitive-behavioral therapy, behavioral weight loss, and sequential treatment for obese patients with binge-eating disorder: a randomized controlled trial. J Consult Clin Psychol 2011;79:675-85.

35. Augustijn MJ, D’Hondt E, Leemans A, Van Acker L, De Guchtenaere A, Lenoir M, et al. Weight loss, behavioral change, and structural neuroplasticity in children with obesity through a multidisciplinary treatment program. Hum Brain Mapp 2019; 40:137-50.

36. Hilbert A, Petroff D, Herpertz S, Pietrowsky R, Tuschen-Caffier B, Vocks S, et al. Meta-analysis of the efficacy of psychological and medical treatments for binge-eating disorder. J Consult Clin Psychol 2019;87:91-105.

37. Pourabbasi A, Larijani B. COGNITOMICS: a new approach for the evaluation of the relationship between diseases and cognition to be designed based on normal behavioral tendencies in real life, a conceptual framework. J Diabetes Metab Disord 2017;16:20. 